\title{
Investigation of Protonation Equilibria and Spectroscopic Properties of the (Aminostyryl)pyridinium Chromophore in Solution, Spread Monolayers, and Langmuir-Blodgett Films
}

\author{
L. F. Chi, ${ }^{*}$ A. Dhathathreyan, and D. Möbius \\ Max-Planck-Institut für biophysikalische Chemie, Postfach 2841, D-3400 Göttingen, \\ West Germany
}

Received May 19, 1989. In Final Form: March 14, 1990

\begin{abstract}
The protonation equilibria of the (aminostyryl)pyridinium chromophore have been investigated in aqueous solutions, in spread monolayers at the air/water interface, and in transferred monolayers on solid substrates (LB films) by measuring absorption, emission, and reflection spectra. The results indicate a $\mathrm{pH}$ shift for the chromophore in monolayers as compared to the aqueous solution. Further, deprotonation of the dye takes place possibly during transfer to solid substrates, especially when the hydrophilic head groups of the dye are oriented away from the hydrophilic surface of the substrate and a protective layer is coated on top. The equilibrium between the neutral and protonated species of the dye is reversibly shifted in LB films by reaction with gas-phase $\mathrm{HCl}$ or $\mathrm{NH}_{3}$ with no discernible degradation in optical quality.
\end{abstract}

\section{Introduction}

(Aminostyryl)pyridinium dyes are used as optical potentiometric tools in electrophysiology. ${ }^{1}$ These dyes display spectroscopic response to membrane potential and have been used extensively for the study and characterization of model membranes, nerve and muscle tissue, organelles, microorganisms, and red blood cells. ${ }^{2-4}$ Due to the wide applications of these dyes as analytical tools, much interest has been devoted to the study of the structure of these dyes in solution as well as in solid state..$^{5}$

The unique properties of the (aminostyryl)pyridinium chromophore suggest that synthetic assemblies of this and related chromophores may be potentially useful as optical devices and as chemical sensors. ${ }^{6}$ Consequently, the elucidation of the structural organization and the spectral properties of these dyes in ordered molecular assemblies are of great interest. In this work, the absorption and fluorescence spectra of such a dye in monolayer at the air/ water interface have been measured. Spectroscopic properties of Langmuir-Blodgett films (LB films) of this dye (dye I, Figure 1) transferred onto solid substrates ${ }^{7}$ have been studied, and the orientation of the chromophores in the monolayer has been evaluated. It is known that the structure, morphology, and interactions of the dye molecules in LB films control at least to some extent the electronic properties of the dye. ${ }^{8}$ Since this dye is not water soluble due to the long hydrocarbon chain, dye II shown in Figure 1, which is similar in structure to dye I, has been used to study the spectral properties of the (aminosturyl). pyridinium chromophore in aqueous solution. Both dyes can exist as a monocation, where one $\mathrm{N}$ in the chromophore is protonated, or as a dication, in which both nitrogen atoms are protonated depending on the $\mathrm{pH}$ of the

(1) Waggoner, A. S. Annu. Rev. Biophys. Bioenerg. 1979, 8, 847

(2) Cohen, L. B.; Salzberg, B. M. Rev. Physiol. Biochem. Pharmacol. $1978,83,35$.

(3) Freedman, J. C.; Laris, P. C. Int. Rev. Cytol. Suppl. 1981, 12, 177

(4) Loew, L. M. Biochem. Biophys. Methods 1982, 6, 243.

(5) Fluhler, E.; Burnham, V. G.; Loew, L. M. Biochemistry 1985, 24, 5749 .

(6) Chi, L. F., Ph.D.Thesis, to be submitted at the University of Göttingen, 1989 .

(7) Blodgett, K. B.; Langmuir, I. Phys. Rev. 1937, 51, 964

(8) Roberts, G. G. Contemporary Phys. 1984, 25, 109.
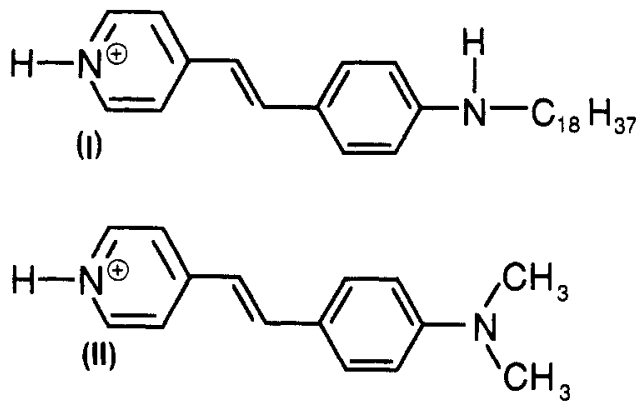

Figure 1. Structures of dye I and dye II.

medium:

$$
\begin{gathered}
\mathrm{DH}_{2}^{2+} \rightleftharpoons \mathrm{DH}^{+}+\mathrm{H}^{+} \\
\mathrm{DH}^{+} \rightleftharpoons \mathrm{D}+\mathrm{H}^{+}
\end{gathered}
$$

\section{Experimental Section}

Materials and Methods. Dyes I and II (E. Merck) were used without further purification. Arachidic acid $\left(C_{20}\right)$ and eicosylamine $\left(\mathrm{C}_{20} \mathrm{NH}_{2}\right)$ used to make $\mathrm{LB}$ films with dye I were obtained from Sigma Chemie. The spreading solvent used was purified $\mathrm{CHCl}_{3}$, and deionized Milli-Q water (Millipore system) was used for preparing the subphase.

Monolayer Preparation. All monolayers were prepared on a rectangular trough made of PTFE provided with a Wilhelmy balance for measuring the surface pressure. The surface potential was measured by using a vibrating plate. For the measurement of surface pressure-molecular area $(\Pi-A)$ and the surface potential-molecular area $(\Delta V-A)$ diagrams, the films were compressed at a constant rate of $5 \AA^{2} /$ molecule per min. They were then transferred onto glass or quartz substrates by standard LB techniques. ${ }^{7}$ The dipping machine used for the monolayer transfer was a modified version of that described earlier. ${ }^{\theta}$ A transfer ratio of $1.0(+0.05)$ was obtained for all monolayers. For the spectroscopic studies of the LB films (see below), the slides were coated on only one side with a single monolayer.

Absorption and Emission Spectroscopy. Absorption and fluorescence spectra of the LB films were measured with the spectrometers described earlier.9 Since the reflectivity of the glass plate is modified by deposited monolayers, the absorp-

(9) Kuhn, H.; Möbius, D.; Bücher, H. Physical Methods of Chemistry; Wiley: New, York, 1972; pp 552-702, I, IIIB. 


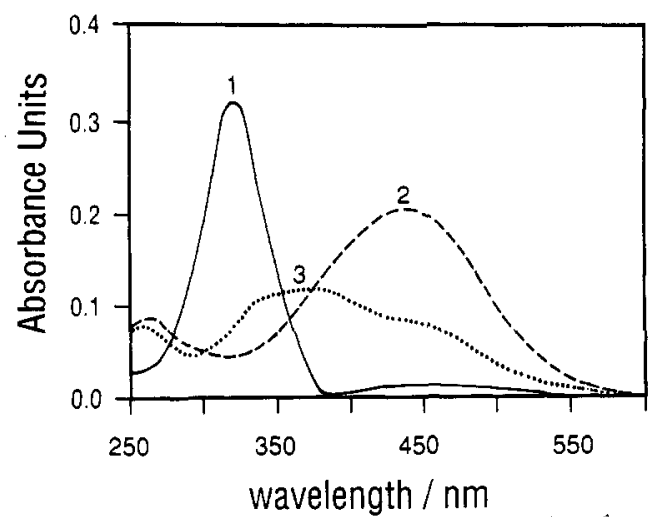

Figure 2. Absorption spectra of dye II (concentration $10^{-5} \mathrm{M}$ ) at different $\mathrm{pH}$ in aqueous solution: (1) at $\mathrm{pH} 2.0$; (2) $\mathrm{pH} 5.6$; (3) $\mathrm{pH} 6.98$.

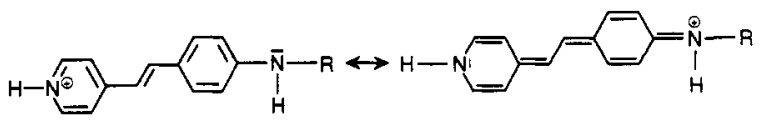

Figure 3. Scheme of two mesomeric structures of dye I.

tion is given here as normalized difference in transmission, $\Delta T$ / $T_{\text {ref }}$, between the reference and the sample areas of the glass plate. $T_{\text {ref }}$ is the transmission of the reference area.

Absorption spectra of dye II in aqueous solutions were recorded with a Shimadzu Model UV-160 spectrometer. The corresponding fluorescence spectra were measured with a PerkinElmer Model LS-5 instrument. The solutions were buffered with Tris- $\mathrm{HCl}$. The reflection spectrometer for measuring reflection spectra of the dye monolayer at oblique incidence at the air/water interface has been described earlier. ${ }^{10,11}$ All the measurements were done at room temperature.

\section{Results and Discussion}

Protonation Equilibrium in Aqueous Solutions. The solubility of dye II depends on the $\mathrm{pH}$ value of the solvent. At acidic $\mathrm{pH}$ of 2.0 , the dye is highly soluble, at pH 5.6 slightly soluble $\left(\sim 7 \times 10^{-6} \mathrm{M}\right)$, and at $\mathrm{pH} 8.0$ insoluble $\left(<10^{-7} \mathrm{M}\right)$. The solutions at different $\mathrm{pH}$ were made up as follows: a concentrated solution of dye II $(0.01$ M) was first made at $\mathrm{pH} 2.0$ by using $\mathrm{HCl}$, and all other solutions were made up to $10^{-5} \mathrm{M}$ by diluting the stock solution with $\mathrm{HCl}$-water for $\mathrm{pH}<4$ and with buffer for higher $\mathrm{pH}$ values.

The absorption spectra of dye II in aqueous solution in its various states of protonation are shown in Figure 2. At pH 2 (curve 1), an absorption maximum is observed at 325 $\mathrm{nm}$, and here the dye is mostly in the form of dication. At pH 5.6 this shifts to $440 \mathrm{~nm}$ (curve 2), and this may be attributed to the monocation. As the $\mathrm{pH}$ is increased beyond 6 , the peak at $440 \mathrm{~nm}$ decreases, and a new band appears between 340 and $400 \mathrm{~nm}$ (curve 3 ). This corresponds then to the neutral species of the dye. It is to be noted here that the band corresponding to the monocation is "red shifted" with respect to the other species. This longer wavelength band is assigned to a transition involving the conjugated $\pi$-electron system and one electron pair on the nitrogen. Due to the electron delocalization, the two mesomeric structures of dye I (shown in Figure 3) are possible. Such electron delocalization can be expected for the monocation form only. Therefore, the

(10) Möbius, D.; Orrit, M.; Grüniger, H.; Meyer, H. Thin Solid Films 1985, 132, 41.

(11) Orrit, M.; Möbius, M.; Lehmann, U.; Meyer, H. J. Chem. Phys. $1986,85,4966$.

(12) Dissociation constants of Organic Bases, IUPAC Suppl. 1972

(13) Israelachvili, J. N. Intermolecular and surface forces; Academic: New York, 1985.

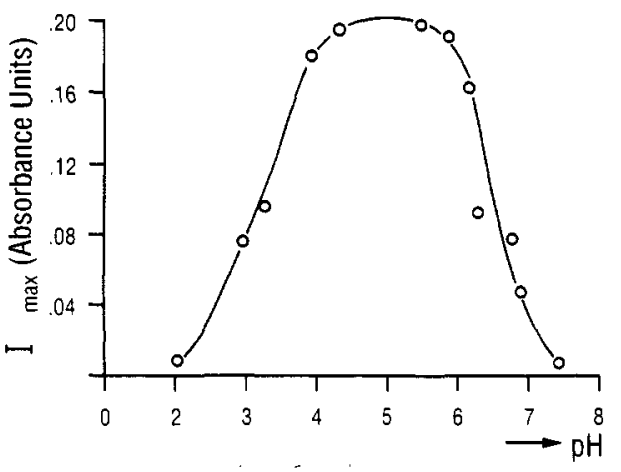

Figure 4. Plot of absorption $I_{\max }(\lambda=440 \mathrm{~nm})$ of dye II solution $\left(10^{-5} \mathrm{M}\right)$ as a function of $\mathrm{pH}$.

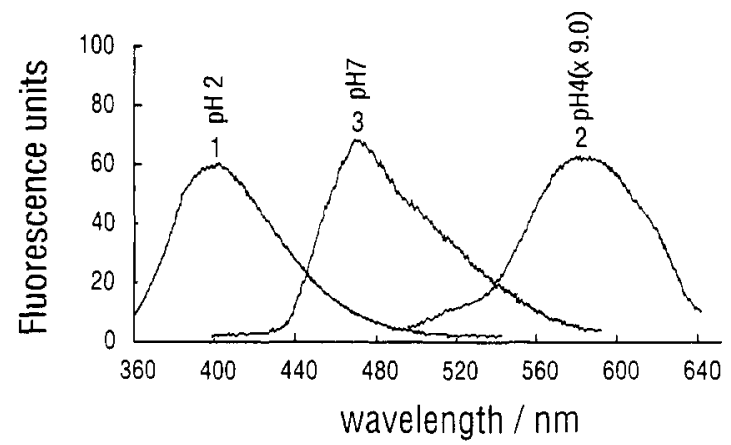

Figure 5. Fluorescence spectra of dye II in aqueous solution at different $\mathrm{pH}$ : (1) $\mathrm{pH} 2.0$; (2) $\mathrm{pH} 4.0$; (3) $\mathrm{pH} 7.0$.

dication and the deprotonated form have shorter wavelength absorption bands.

On the basis of the proportionality between the absorption intensity and the fraction of protonated species, one can determine the $\mathrm{p} K$ value of dye II by $\log [\alpha /(1-$ $\alpha)]=\mathrm{pH}-\mathrm{p} K_{\mathrm{a}}$ from the dependence of $I_{\max }$ of the monocation (i.e., at $440 \mathrm{~nm}$ ) on $\mathrm{pH}$ (shown in Figure 4). Here $\alpha$ is the ratio of absorption $I$ at $\lambda=440 \mathrm{~nm}$ to $I_{0}$, the absorption of the monocation in the plateau region from Figure 4. This evaluation can be done since both the dicationic and the neutral forms do not absorb at $440 \mathrm{~nm}$ as seen from the absorption values in Figure 4 at $\mathrm{pH} 2$ and $\mathrm{pH}$ 7.5. A plot of $\log [\alpha /(1-\alpha)]$ as a function of $\mathrm{pH}$ yielded $\mathrm{p} K$ values of 3.2 and 6.7 for dye II. These values are in reasonable agreement with the $\mathrm{p} K$ values for aniline (4.69) and substituted pyridine (5.28).

The fluorescence emission spectra for the various protonation states of dye II are shown in Figure 5. The fluorescence band with the maximum at $580 \mathrm{~nm}$ obtained for $\mathrm{pH} 4.0$ corresponds to the monocation (curve 2). A blue shift occurs in going from $\mathrm{pH} 4.0$ to neutral media. When the dye is neutral at $\mathrm{pH} 7.0$, the maximum occurs at 470 $\mathrm{nm}$ with a shoulder at about $500 \mathrm{~nm}$. The dication shows an emission maximum at $410 \mathrm{~nm}(\mathrm{pH} \mathrm{2})$. From the intensities of the respective spectra, it is seen that the strongest fluorescence is observed for the neutral species.

Protonation Equilibria in Monolayers. Dye I has the same chromophore as dye II. It is not soluble in water because of the long hydrocarbon chain. The pure dye can form stable monolayers. The area required for a dye molecule in this layer at $20 \mathrm{mN} / \mathrm{m}$ is $25 \AA^{2}$. Since the dye in the pure monolayer tends to aggregate, mixed monolayers of the dye with $\mathrm{C}_{20}$ in the molar ratio $1: 5$ were used to perform the monolayer experiments. The mixed dye/ $\mathrm{C}_{20}$ solution was spread on an aqueous subphase. In Figure 6 the surface pressure $\Pi$ of the mixed film is plotted against the area per molecule together with the surface potential $\Delta V$. The area/molecule of the dye at a surface pressure 


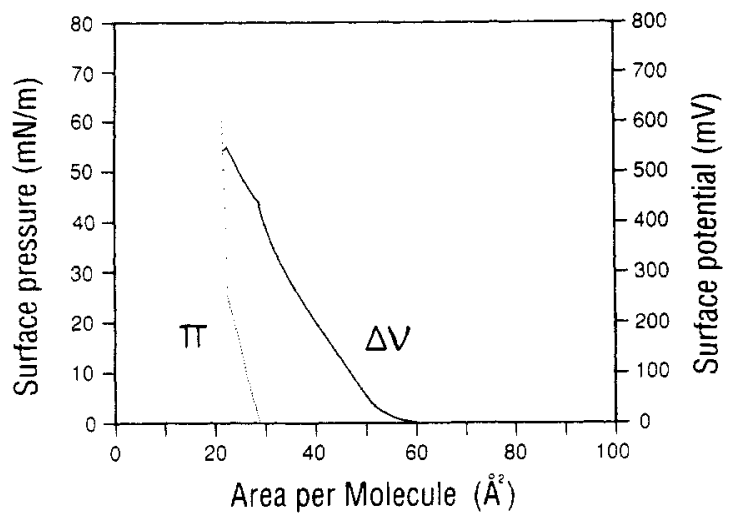

Figure 6. Surface pressure $\Pi$ and surface potential $\Delta V$ plotted against molecular area for dye I in the mixed monolayer with arachidic acid, molar ratio 1:5 (pH 5.6).

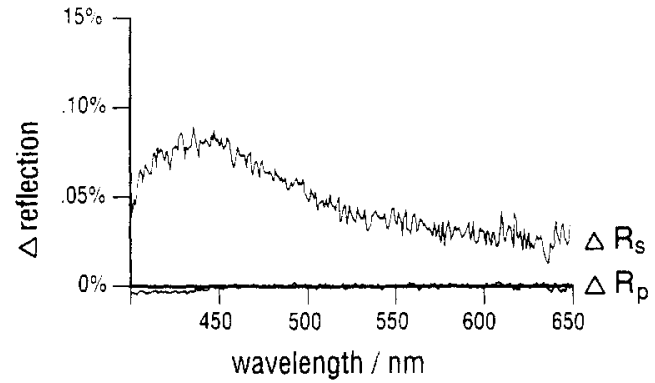

Figure 7. Reflection spectra of a mixed monolayer of dye $I$ and arachidic acid, molar ratio 1:5, at the air/water interface, $\mathrm{pH} 5.6$, surface pressure $20 \mathrm{mN} / \mathrm{m}$.

of $20 \mathrm{mN} / \mathrm{m}$ is $18.5 \AA^{2}$. The decrease in area of the dye in the mixed layer is evidence for a homogeneous mixing of the dye in the matrix.

The protonation of the dye at the air/water interface depends on the $\mathrm{pH}$ of the subphase, and the protonation of dye I at different $\mathrm{pH}$ at the air/water interface can be studied by using the reflection spectroscopic technique. ${ }^{10,11}$ Figure 7 shows the reflection spectra of dye I at pH 5.6 measured at oblique incidence $\left(45^{\circ}\right)$ using polarized light. The spectra show a band with a maximum at $450 \mathrm{~nm}$ for perpendicularly polarized light $\left(\Delta R_{\mathrm{s}}\right)$ and a small negative band for parallel polarized light $\left(\Delta R_{p}\right)$. It is seen that the spectrum of dye $I$ at the air/water interface is bathochromically shifted compared to that in $\mathrm{CHCl}_{3}$ solution $\left(\lambda_{\max }\right.$ $=364 \mathrm{~nm}$ ). This effect could be explained on the basis of the protonation of the dye (see below). By determining the orientation parameter $P^{10,11}$ obtained by using the ratio $\Delta R_{\mathrm{p}} / \Delta R_{\mathrm{B}} \approx 0$ (at $\left.450 \mathrm{~nm}\right)(P=1 / 3, \vartheta=55)$, it is seen that the chromophores of dye I are oriented spatially statistically at the air/water interface. These spectra agree quite well with the solution spectra obtained for dye II at pH 5.6, indicating that dye I exists in its monocation form at the air/water interface. Since the spectrum of dye I may depend on the local electrical potential, the reflection spectrum was also measured in the presence of $10^{-3} \mathrm{M}$ $\mathrm{NaCl}$. No difference to the spectrum obtained in the absence of $\mathrm{NaCl}$ was observed. At a subphase $\mathrm{pH}$ of 2.0 , the spectrum corresponding to the monocation is retained, and no new band attributed to the dication is obtained. This is reasonable since the approach of the second proton to the charged interface requires more energy than the uptake of the second proton by the monocation in solution. At $\mathrm{pH}$ 9.35, the reflection spectrum of dye I shows a band with a maximum at $350 \mathrm{~nm}$ which corresponds to the neutral form. It is seen here that monolayers at the airwater interface a higher $\mathrm{pH}$ is necessary to obtain the
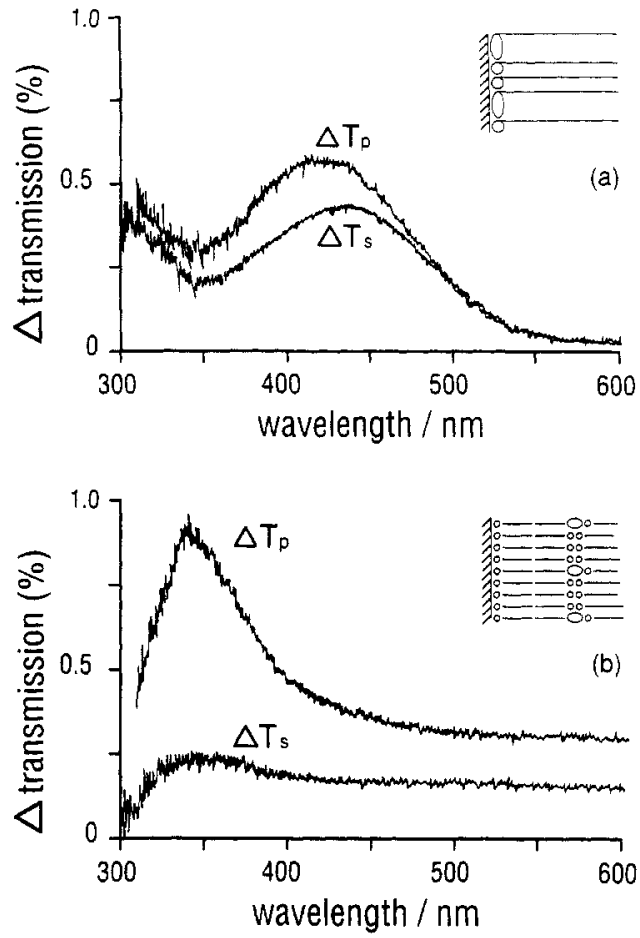

Figure 8. Transmission spectra of a mixed monolayer of dye I

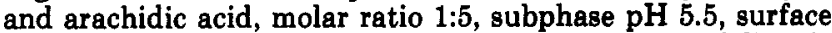
pressure at transfer $20 \mathrm{mN} / \mathrm{m}$ : (a) monolayer transferred directly to glass; (b) monolayer transferred to hydrophobic surface.

neutral form than with dye II in solution since the $\mathrm{p} K_{\mathrm{a}}$ values are different.

Protonation Equilibria in Transferred Monolayers (LB Films). Figure 8 shows the UV-vis absorption spectra of Langmuir-Blodgett films (LB films) of dye I transferred to glass plates with the top (a) corresponding to the case when the LB films are transferred directly onto glass and the bottom to when the films are transferred to a substrate hydrophobized previously by coating with one layer of $\mathrm{C}_{20}$ or $\mathrm{C}_{20} \mathrm{NH}_{2}$ or by treating the glasses with a $5 \%$ solution of dimethyldichlorosilane in chloroform. The two spectra correspond to the transmission spectra measured at an angle of incidence of $45^{\circ}$ with the electric vector polarized parallel $\left(\Delta T_{\mathrm{p}}\right)$ and perpendicular $\left(\Delta T_{\mathrm{g}}\right)$ to the plane of incidence. The peak positions for $\Delta T_{\mathrm{s}}$ and $\Delta T_{\mathrm{p}}$ in Figure 8 lie around $430 \mathrm{~nm}$ close to the absorption maxima for dye II between $\mathrm{pH} 4$ and 6 . This corresponds to the monocation form of the dye in solution and also to that of the dye monolayer at the air/water interface at $\mathrm{pH}$ 5.6. The values of $\Delta T_{\mathrm{s}} / \Delta T_{\mathrm{p}}$ provide the orientation parameter of the transition moments $(P=0.28$ for Figure $8 \mathrm{a}$ and $P=0.76$ for Figure $8 \mathrm{~b})$. This indicates a better orientation of the transition dipole moments of the dye I in the LB film in Figure $8 \mathrm{~b}$ (angle between transition moment and surface normal $\vartheta=58^{\circ}$ and $\vartheta=29^{\circ}$ for Figure $8 \mathrm{a}$ and $8 \mathrm{~b}$, respectively) compared to the arrangement shown in the inset, Figure $8 \mathrm{a}$. The spectra of the LB films of dye I transferred onto hydrophobic substrate (Figure 8b) show a blue shift, and the peak positions are now at $350 \mathrm{~nm}$. This corresponds to the solution spectra of dye II measured in chloroform, where the dye exists in its neutral form. The corresponding fluorescence spectra also show similar changes to red and green species for the above two cases (spectra not shown here). Since the absorption of dyes like dye I in solution depends on the polarity, the spectrum of dye II was measured in chloroform and in propanol solution. Only a difference of $10 \mathrm{~nm}$ in peak position was observed. Therefore, the assignment of the bands in analogy to the 
situation of dye I in solution is correct. Further, the environment of the chromophore of dye I in transferred monolayers was changed by deposition of monolayers with different head groups $\left(\mathrm{NH}_{3}{ }^{+}, \mathrm{OH}\right.$, and $\left.\mathrm{COOCH}_{3}\right)$ on top of the monolayer with dye I. No influence of head group on the spectrum of dye I was observed. This is true also for a variation of the dipole layer constituted by the hydrophobic end groups of the monolayers ${ }^{14}$ at a distance of $2.5 \mathrm{~nm}$ from the dye chromophores influencing the electrostatic potential at the chromophore. Therefore, polarity effects do not affect the assignment of the bands to the different forms of the chromophore.

The reflection spectra at the air/water interface at $\mathrm{pH}$ 5.6 measured for dye I show the monocation form whereas LB films of the dye transferred to hydrophobic surface show the spectra corresponding to the neutral species. Therefore, a deprotonation of the dye takes place during the transfer of the monolayer. One reason for this behavior may be the following: after transfer of the monolayers to a hydrophobic surface, the head groups of the dye monolayers are still in contact with water and therefore are hydrated. Subsequently, the dye monolayer is coated with a protective layer of $\mathrm{C}_{20}$ or $\mathrm{C}_{20} \mathrm{NH}_{2}$ in order to remove the glass plates from the water. During transfer of the protective layer to the hydrated head groups of the monolayer, electrostatic and hydration forces undergo changes. ${ }^{13}$ The magnitude of these forces is reduced when the dye carries no charge or is in its neutral form. Further, it is well-known that interfacial $\mathrm{pH}$ differs from that of the bulk. ${ }^{15}$

In the case of the dye monolayer in direct contact with the glass substrate (Figure 8a), the polar hydroxy groups on the substrate stabilize the polar groups of the monolayer, and the monocation form is retained. It is also seen that the state of deprotonation when dye I is transferred at $\mathrm{pH} 7.9$ is retained by the glass substrate. The equilibrium between the protonated and neutral species can be reversibly shifted by exposing the $\mathrm{LB}$ films to $\mathrm{HCl}$ or $\mathrm{NH}_{4} \mathrm{OH}$ vapor, respectively (Figure 9 ). It is possible to partially protonate chromophores of dye I transferred to hydrophobic surface (absorption maxima in blue) by exposing the $\mathrm{LB}$ film to $\mathrm{HCl}$ vapor. The monocation in the LB film (absorption maxima in red) could be deprotonated by exposing it to $\mathrm{NH}_{3}$ vapor above an aqueous

(14) Möbius, D.; Cordroch, W.; Loschek, R.; Chi, L. F.; Dhathahtreyan A.; Vogel, V. Thin Solid Films 1989, 179, 53.

(15) Davies, J. T.; Rideal, E. K. Interfacial Phenomena; Academic: New York, 1963

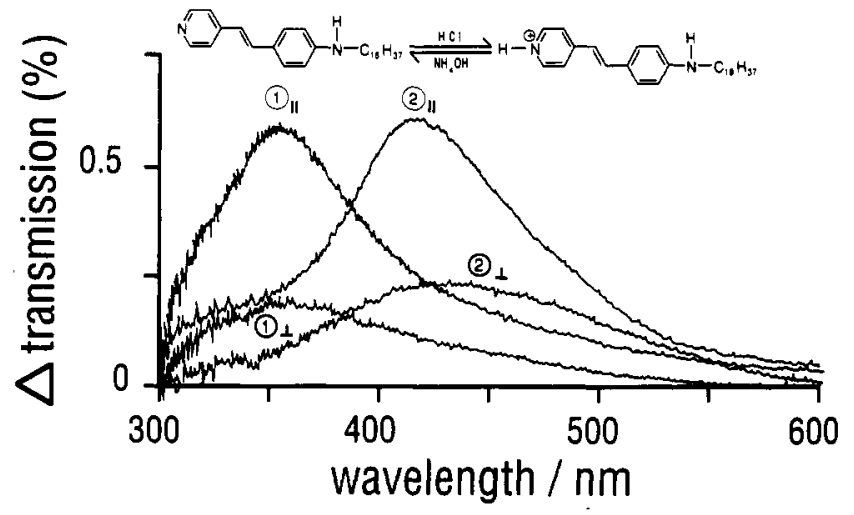

Figure 9. Transmission spectra of dye I: (1) mixed monolayer of dye I with arachidic acid (molar ratio 1:5) on hydrophobic surface; (2) after exposure to $\mathrm{HCl}$.

$\mathrm{NH}_{4} \mathrm{OH}$ solution. The shift in the protonation equilibrium is dependent on the time of exposure, and the preferential orientation of the monolayer was reduced on long exposure to concentrated acid. It is known that the monocationic chromophore is useful in electrooptic measurements and shows high nonlinear hyperpolarizabilities ${ }^{6}$ because of its strong noncentrosymmetric structure in comparison to the neutral form. Repeated cycling between the "blue" and "red" forms under mild conditions in the vapor phase resulted in negligible degradation of film quality.

\section{Conclusion}

The protonation equilibria of the (aminostyryl)pyridinium chromophore in the LB film depends on the hydrophilic environment of the monolayer. The chromophores of the dye have a spatial statistical orientation at the air/water interface and take a preferential orientation in LB film with the transition moments oriented nearly perpendicular to the layer plane. The protonation equilibria can be manipulated in the LB films without damaging the monolayer and without change of the orientation of the dye chromophores. Such LB films are of interest in the construction of electronic or optical devices as molecular switches using monomolecular layers of dyes.

Acknowledgment. This work was funded by the Bundesministerium für Forschung and Technologie (No. 038620 ). We thank Dr. G. Marowsky for providing the dyes used in this work. 